\title{
An Effective Modeling Framework for the Analysis of Interconnects Subject to Line-Edge Roughness
}

\author{
Paolo Manfredi, Member, IEEE, Dries Vande Ginste, Senior Member, IEEE, Daniël De Zutter, Fellow, IEEE
}

\begin{abstract}
This letter proposes a complete and efficient simulation framework to assess the effects of line-edge roughness appearing in on-chip lines. The modeling approach consists of three steps. First, a stochastic macromodel is created for the per-unit-length RLGC parameters of the line. Secondly, random conductor edge profiles are generated using randomized splines. These are combined with the stochastic macromodel to readily provide place-dependent RLGC parameters. Finally, the resulting nonuniform transmission line is analyzed by means of a fast and accurate perturbation technique. To validate the proposed approach, a statistical analysis of the response of a coupled inverted embedded microstrip line is carried out for different roughness parameters.
\end{abstract}

Index Terms-Line-edge roughness, nonuniform transmission lines, on-chip interconnects, perturbation methods.

\section{INTRODUCTION}

The increasing shrinking and miniaturization of device sizes is leading to a non-negligible impact of line-edge roughness (LER) on mm-wave and nanoscale on-chip interconnects [1]. LER is introduced by several sources in the manufacturing process, including photolitographic mask uncertainties and chemical properties of resist [2]. Owing to the inherent random nature of the problem, several statistical approaches have been proposed for the determination of the resistance and/or capacitance of on-chip lines [3]-[5]. The aforementioned studies rely on a statistical representation of the LER geometry, ranging from simple sinusoidal models to correlated Gaussian processes. However, a general and comprehensive method to assess the impact of LER on the global interconnect performance, including all wave effects [6], appears yet to be missing.

This letter addresses this issue by putting forward a new modeling framework for the electrical performance of onchip lines subject to LER, which is summarized as follows. First, a stochastic macromodel of the per-unit-length (p.u.1.) RLGC parameters, accounting for conductor edge variations, is constructed by using polynomial chaos expansions (PCEs) [7]. The p.u.l. parameters are obtained under the quasi-TM field assumption with a boundary integral equation solver [8] capable of accurately capturing skin and slow-wave effects, which unavoidably appear at the frequencies of interest. To limit the computationally expensive calls to this electromagnetic field solver, the calculation of the PCE coefficients is carried out by

Manuscript received February 03, 2015; revised April 16, 2015; accepted May 16, 2015. This work was funded by the Research Foundation Flanders (FWO-Vlaanderen). P. Manfredi is an FWO Postdoctoral Research Fellow.

P. Manfredi, D. Vande Ginste and D. De Zutter are with the Electromagnetics Group, Department of Information Technology, Ghent University/iMinds, 9000 Gent, Belgium (e-mail: paolo.manfredi@ugent.be, dries.vandeginste@ugent.be, daniel.dezutter@ugent.be). applying a stochastic testing (ST) method [9]. Second, rough conductor edge profiles are generated by means of randomized splines. The tuning of the spline parameters allows to achieve different roughness characteristics. Once the trace profiles are known, the stochastic macromodel is used to conveniently obtain the place-dependent RLGC p.u.l. parameters. Finally, a perturbation technique (PT) [10] is used to efficiently calculate the S-parameters of the resulting, inherently nonuniform transmission line (NUTL), as the methodology in [7] applies to uniform lines only.

\section{Proposed Modeling Framework}

For the sake of illustration, the discussion is based on the coupled inverted embedded micristrip (IEM) lines shown in Fig. 1, where all the relevant geometrical and material parameters are indicated. The line has two aluminum signal conductors embedded in a silicon dioxide layer lying on a silicon substrate, and an aluminum ground on top. The generalization to lines with different geometries and/or number of conductors is straightforward.

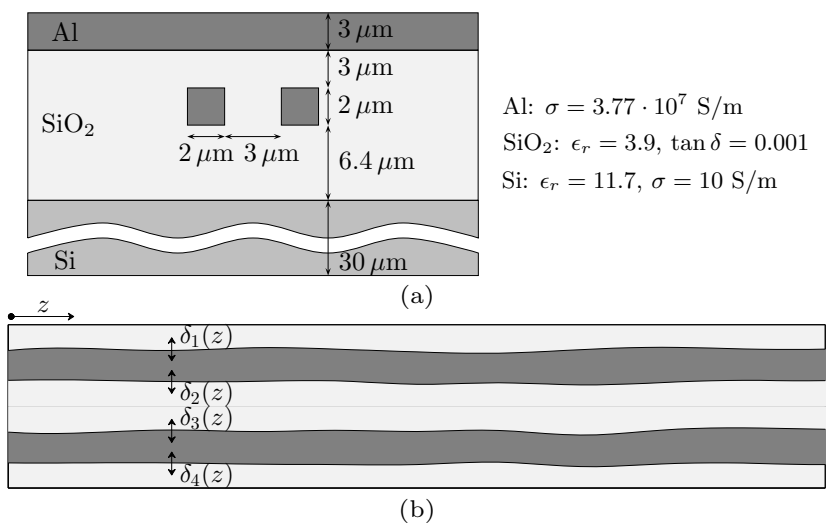

Fig. 1. Coupled IEM lines: (a) nominal cross-section; (b) top view of the signal conductors with LER.

As shown in Fig. 1(b), in the presence of LER, the actual position of the conductor edges varies along the direction of propagation $z$. A local deviation $\delta_{i}(z)(i=1,2,3,4)$ from the nominal position is introduced for each of the four edges. The structure is therefore a NUTL, whose behavior is described by the following set of Telegrapher's equations [11]

$$
\begin{aligned}
& \frac{d}{d z} \mathbf{V}(\omega, z)=-j \omega \mathcal{L}(\omega, z) \mathbf{I}(\omega, z) \\
& \frac{d}{d z} \mathbf{I}(\omega, z)=-j \omega \mathcal{C}(\omega, z) \mathbf{V}(\omega, z)
\end{aligned}
$$

where $\omega$ is the angular frequency, whilst $\mathbf{V}=\left[V_{1}, V_{2}\right]^{T}$ and $\mathbf{I}=\left[I_{1}, I_{2}\right]^{T}$ collect the voltages and currents along the two 
conductors. The quantities

$$
\begin{aligned}
& \mathcal{L}(\omega, z)=\mathbf{L}\left(\omega, \delta_{1}(z), \ldots, \delta_{4}(z)\right)+\mathbf{R}\left(\omega, \delta_{1}(z), \ldots, \delta_{4}(z)\right) / j \omega \\
& \mathcal{C}(\omega, z)=\mathbf{C}\left(\omega, \delta_{1}(z), \ldots, \delta_{4}(z)\right)+\mathbf{G}\left(\omega, \delta_{1}(z), \ldots, \delta_{4}(z)\right) / j \omega
\end{aligned}
$$

are the frequency- and place-dependent complex p.u.l. inductance and capacitance matrices, which are functions of the RLGC parameters evaluated for the pertinent configuration of the conductor geometry at a given position $z$. Possible correlation in the edge variations can be readily accounted for by means of principal component analysis [12].

\section{A. Stochastic Macromodel of the RLGC Parameters}

Assuming a Gaussian distribution for the variations $\delta_{i}$, the p.u.l. parameters are approximated by means of the following Hermite-PCE [7]

$$
\mathcal{X}(\omega) \approx \sum_{k=1}^{K} \mathcal{X}_{k}(\omega) \varphi_{k}\left(\xi_{1}, \xi_{2}, \xi_{3}, \xi_{4}\right),
$$

where $\mathcal{X}$ stands for any of the p.u.l. parameter matrices $\mathbf{R}, \mathbf{L}$, $\mathbf{G}$ and $\mathbf{C}$, while $\xi_{i}=\delta_{i} / \sigma_{r}$ is the edge variation normalized w.r.t. the absolute standard deviation $\sigma_{r}$ of the roughness. The functions $\varphi_{k}\left(\xi_{1}, \xi_{2}, \xi_{3}, \xi_{4}\right)$ are the quadrivariate Hermite polynomials, which provide the best convergence for the Gaussian distribution of the parameters. Note that other statistical distributions can be treated in a similar way.

In order to limit the computational burden, the PCE coefficients $\mathcal{X}_{k}(\omega)$ are calculated via ST [9], i.e., by point matching the expansion (3) at a pre-defined set of $K$ points $\left\{\left(\xi_{1}, \xi_{2}, \xi_{3}, \xi_{4}\right)_{m}\right\}_{m=1}^{K}$ and inverting the resulting system:

$$
\left[\begin{array}{c}
\mathcal{X}_{1}(\omega) \\
\vdots \\
\mathcal{X}_{K}(\omega)
\end{array}\right]=\left(\mathbf{A}^{-1} \otimes \mathbb{I}\right)\left[\begin{array}{c}
\check{\mathcal{X}}_{1}(\omega) \\
\vdots \\
\check{\mathcal{X}}_{K}(\omega)
\end{array}\right] \forall \omega .
$$

In the above equation, $\check{\mathcal{X}}_{m}$, with $m=1, \ldots, K$, is the p.u.l. parameter sampled with the field solver [8] at the $m$ th ST point, $\mathbf{A}$ is a $K \times K$ matrix with entries $A_{m k}=$ $\varphi_{k}\left(\xi_{1 m}, \xi_{2 m}, \xi_{3 m}, \xi_{4 m}\right)$, i.e., the $k$ th polynomial evaluated at the same point, whilst II is the identity matrix of the same size as the number of rows of $\mathcal{X}$. The ST points are generated with a suitable algorithm [9]. This approach limits the calls to the field solver to $K$ times only, in contrast to other approaches that require a much higher number of samples to solve for the PCE coefficients.

\section{B. Line-Edge Roughness Model}

The PCE (3) provides a compact macromodel for the p.u.l. parameters as a function of the edge variations. To model LER, a suitable description for the longitudinal behavior of $\delta_{1,2,3,4}(z)$ is required. The proposed idea is to subdivide the line length $\ell$ in a given number of points $N_{r}$, where the variations of the conductor edges are assumed to be mutually independent. Samples for these variations are then drawn according to a Gaussian distribution. The values at intermediate positions are obtained by cubic spline interpolation, thus ensuring a sufficient smoothness of the edge profile. The separation $\Delta \ell=\ell /\left(N_{r}-1\right)$ between two independent points can be interpreted as a correlation distance. By tuning the standard deviation $\sigma_{r}$ of the geometrical parameters, as well as the correlation distance $\Delta \ell$, the roughness profile can be adjusted to match measured data.

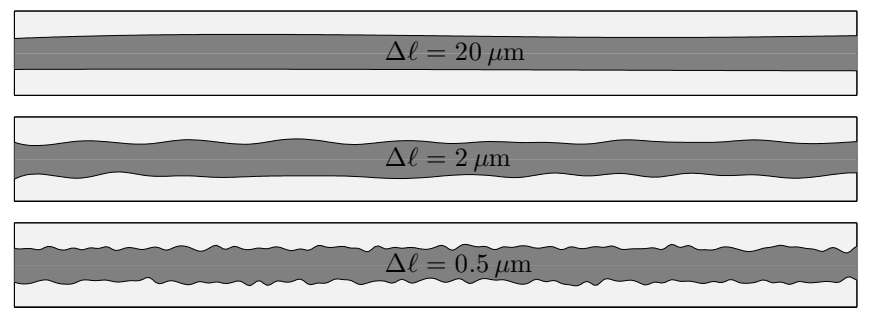

Fig. 2. IEM conductor profiles obtained with different values of $\Delta \ell$, displayed with scales preserved over a section of $50 \mu \mathrm{m}$.

Different LER profiles are combined to reproduce the longitudinal shape of the conductors. For example, Fig. 2 shows three different profiles (with the axis aspect ratio preserved) for the conductors of one of the IEM lines of Fig. 1, obtained with a standard deviation of $\sigma_{r}=0.1 \mu \mathrm{m}$ and different values of $\Delta \ell$. The picture shows how the "degree of nonuniformity" indeed varies with the parameter $\Delta \ell$. Moreover, it is intuitively understood that increasing the standard deviation results in the edge to be rougher. Additionally, Fig. 1(b) depicts the profiles of the two coupled lines obtained with $\Delta \ell=5 \mu \mathrm{m}$. These profiles are in good agreement with real profiles measured with scanning electron microscopes (see, e.g., [2]). It is important to point out that, besides this alternative proposed approach, state-of-the-art models can be fitted in the proposed simulation framework as well. Once the $z$-dependent LER profiles are available, the PCE (3) is evaluated to inexpensively obtain the place-dependent p.u.l. parameters.

\section{Perturbation Technique (PT) for Nonuniform Lines}

The assessment of the LER impact still requires the solution of the NUTL (1), for which no exact closed-form solution exists. The standard approach to deal with (1) is to discretize the line into short and locally uniform sections and then to calculate the overall chain-parameter matrix (CPM) as the product of the matrices of each section [11]. Unfortunately, this method leads to large computational times and its application hinders the analysis of large sample sizes, e.g., with the aim of assessing the impact of LER from a statistical standpoint. Moreover, the CPM inherently leads to an undesired staircasing of the LER profiles.

To overcome the aforementioned issues, a two-step PT for NUTLs [10], which was proven to be fast and accurate [13], is adopted here. The method is based on the representation of the actual voltages and currents as the sum of three contributions, i.e., $\mathbf{V}=\mathcal{V}_{0}+\mathcal{V}_{1}+\mathcal{V}_{2}$ and $\mathbf{I}=\mathcal{I}_{0}+\mathcal{I}_{1}+\mathcal{I}_{2}$. Each contribution is obtained as the solution of a uniform transmission line with distributed source terms:

$$
\begin{aligned}
& \frac{d}{d z} \mathcal{V}_{i}(\omega, z)=-j \omega \tilde{\mathcal{L}}(\omega) \mathcal{I}_{i}(\omega, z)+\mathcal{V}_{F, i}(\omega, z) \\
& \frac{d}{d z} \mathcal{I}_{i}(\omega, z)=-j \omega \tilde{\mathcal{C}}(\omega) \mathcal{V}_{i}(\omega, z)+\mathcal{I}_{F, i}(\omega, z)
\end{aligned}
$$


$i=0,1,2$, where the constant p.u.l. complex inductance and capacitance matrices are the average of the actual quantities over $z$, i.e., $\tilde{\mathcal{L}}(\omega)=\frac{1}{\ell} \int_{0}^{\ell} \mathcal{L}(\omega, z) d z$ and $\tilde{\mathcal{C}}(\omega)=$ $\frac{1}{\ell} \int_{0}^{\ell} \mathcal{C}(\omega, z) d z$, respectively. Given the source terms and the boundary conditions at the line terminations, the solution of (5) is readily calculated [11]. Specifically, the zeroth-order contributions $\mathcal{V}_{0}$ and $\mathcal{I}_{0}$ are the solution of (5) with null excitation terms (i.e., $\mathcal{V}_{F, 0}=\mathcal{I}_{F, 0}=0$ ). The first- and secondorder contributions are the solution of (5) with forcing terms

$$
\begin{aligned}
& \mathcal{V}_{F, i}(\omega, z)=-j \omega[\mathcal{L}(\omega, z)-\tilde{\mathcal{L}}(\omega)] \mathcal{I}_{i-1}(\omega, z) \\
& \mathcal{I}_{F, i}(\omega, z)=-j \omega[\mathcal{C}(\omega, z)-\tilde{\mathcal{C}}(\omega)] \mathcal{V}_{i-1}(\omega, z)
\end{aligned}
$$

$i=1,2$, instead. Semi-analytical expressions are available for each voltage and current contribution [10].

\section{NUMERICAL RESULTS AND DISCUSSION}

The proposed framework is used to analyze the pair of coupled IEM lines of Fig. 1 with a length $\ell=1 \mathrm{~mm}$.

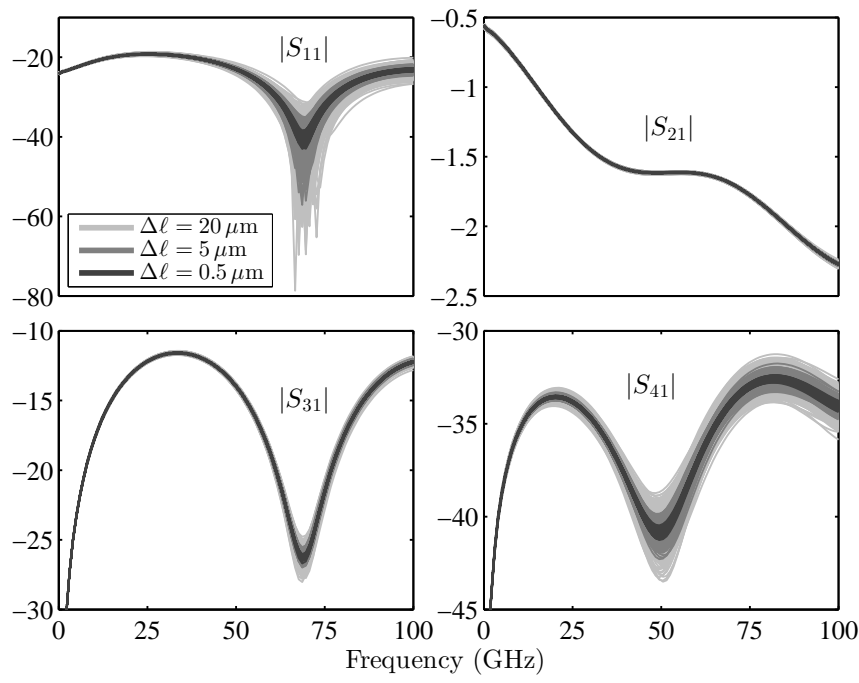

Fig. 3. Magnitudes of the S-parameters (in dB) for the IEM line of Fig. 1, computed for random LER profiles with different correlation distances.

First, a stochastic macromodel consisting of a third-order PCE with $K=35$ terms is created. Next, random LER profiles are generated assuming a standard deviation of $\sigma_{r}=0.1 \mu \mathrm{m}$. Fig. 3 shows the $S$-parameters obtained from the total voltages and currents at the line ends $(z=0$ and $z=\ell)$, calculated for $50 \Omega$ terminations as the sum of the three contributions that are governed by (5). Each group of curves corresponds to 1000 Monte Carlo realizations of LER profiles with a different value of $\Delta \ell$. The darker lines are associated to shorter correlation distances. This study shows that in the case of short correlation distances, the effect of LER tends to be averaged out, thus yielding a smaller variation of the response. This is in agreement with literature results [3]-[5].

The adoption of the PT allows to solve (1) up to $24 \times$ faster than the reference CPM approach, while providing an absolute error on the S-parameters below $2.2 \times 10^{-4}$ (i.e., $-73 \mathrm{~dB}$ ) in magnitude. For further discussion on the accuracy of the PT, the reader is referred to [10] and [13]. The creation of the stochastic macromodel requires about $175 \mathrm{~min}$ instead. It should be noted that this step is essential, as each call to the field solver takes about $5 \mathrm{~min}$. Hence, ad-hoc calculation of many place-dependent p.u.l. parameters via the field solver is virtually impossible! Yet, in contrast to brute-force Monte Carlo approaches, the combination of the stochastic macromodel and the PT makes the statistical analysis feasible. From the calculated responses, statistical information such as quantiles or probability distribution can be extracted as well.

\section{CONCLUSIONS}

An effective modeling framework for on-chip interconnects affected by LER is presented in this letter. The methodology relies on the construction of a PCE-based stochastic macromodel for the RLGC parameters of the line, the generation of random LER profiles via cubic spline interpolation, and the simulation of the resulting NUTL by means of a PT. The combination of the above elements makes the statistical assessment of the interconnect response feasible, accurate and efficient.

\section{REFERENCES}

[1] M. Stucchi, M. Bamal, and K. Maex, "Impact of line-edge roughness on resistance and capacitance of scaled interconnects," Microelectronic Eng., vol. 84, no. 11, pp. 2733-2737, Nov. 2007.

[2] K. Patel, S. N. Lahiri, and C. J. Spanos, "Robust estimation of line width roughness parameters," J. Vacuum Sci. Technology B, vol. 28, no. 6, pp. 18-33, Nov. 2010.

[3] T. Kurusu, H. Tanimoto, M. Wada, A. Isobayashi, A. Kajita, N. Aoki, and Y. Toyoshima, "Impact of line-edge roughness on electrical resistivity in decananoscale copper wires: a Monte Carlo study," in Proc. Int. Conf. on Simulation of Semiconductor Processes and Devices, Denver, CO, USA, Sep. 2012, pp. 304-307.

[4] W. Yu, Q. Zhang, Z. Ye, and Z. Luo, "Efficient statistical capacitance extraction of nanometer interconnects considering the on-chip line edge roughness," Microelectronics Rel., vol. 52, no. 4, pp. 704-710, Apr. 2012.

[5] F. J. Twaddle, D. R. S. Cumming, S. Roy, A. Asenov, and T. D. Drysdale, "RC variability of short-range interconnects," in Proc. Int. Workshop on Computational Electron., Beijing, China, May 2009, pp. 1-3.

[6] B. Curran, I. Ndip, S. Guttowski, and H. Reichl, "A methodology for combined modeling of skin, proximity, edge, and surface roughness effects," IEEE Trans. Microw. Theory Techn., vol. 58, no. 9, pp. 24482455, Sep. 2010.

[7] D. Vande Ginste, D. De Zutter, D. Deschrijver, T. Dhaene, P. Manfredi, and F. G. Canavero, "Stochastic modeling-based variability analysis of on-chip interconnects," IEEE Trans. Compon. Packag. Manuf. Techol., vol. 2, no. 7, pp. 1182-1192, Jul. 2012.

[8] T. Demeester and D. De Zutter, "Quasi-TM transmission line parameters of coupled lossy lines based on the Dirichlet to Neumann boundary operator," IEEE Trans. Microw. Theory Techn., vol. 56, no. 7, pp. 16491660, Jul. 2008.

[9] Z. Zhang, T. A. El-Moselhy, I. M. Elfadel, and L. Daniel, "Stochastic testing method for transistor-level uncertainty quantification based on generalized polynomial chaos," IEEE Trans. Comput.-Aided Des. Integr. Circuits Syst., vol. 32, no. 10, pp. 1533-1545, Oct. 2013.

[10] M. Chernobryvko, D. De Zutter, and D. Vande Ginste, "Nonuniform multiconductor transmission line analysis by a two-step perturbation technique," IEEE Trans. Compon. Packag. Manuf. Techol., vol. 4, no. 11, pp. 1838-1846 , Nov. 2014.

[11] C. R. Paul, Analysis of Multiconductor Transmission Lines, 2nd ed. Hoboken, NJ: Wiley, 2008.

[12] J. S. Ochoa and A. C. Cangellaris, "Random-space dimensionality reduction for expedient yield estimation of passive microwave circuits," IEEE Trans. Microw. Theory Techn., vol. 61, no. 12, pp. 4313-4321, Dec. 2013

[13] M. Chernobryvko, D. Vande Ginste, and D. De Zutter, "A two-step perturbation technique for nonuniform single and differential lines," IEEE Trans. Microw. Theory Techn., vol. 61, no. 5, pp. 1758-1767, May 2013. 